\title{
Linx
}

Revue des linguistes de l'université Paris X Nanterre

70-71| 2014

Variations sémantiques et syntaxiques : aspects d'une théorie de l'invariance

\section{Prolifération et reformulation comme traces de l'activité énonciative à travers l'agencement des formes}

Jean-Jacques Franckel

\section{OpenEdition}

\section{Journals}

Édition électronique

URL : http://journals.openedition.org/linx/1579

DOI : $10.4000 / \operatorname{linx} .1579$

ISSN : 2118-9692

\section{Éditeur}

Presses universitaires de Paris Nanterre

Édition imprimée

Date de publication : 1 septembre 2014

Pagination : 197-207

ISSN : 0246-8743

\section{Référence électronique}

Jean-Jacques Franckel, « Prolifération et reformulation comme traces de l'activité énonciative à travers l'agencement des formes », Linx [En ligne], 70-71 | 2014, mis en ligne le, consulté le 03 mai 2019. URL : http://journals.openedition.org/linx/1579; DOI : 10.4000/linx.1579 


\section{Prolifération et reformulation comme traces de l'activité énonciative à travers l'agencement des formes}

Jean-Jacques Franckel, Université Paris-Ouest Nanterre la Défense

\section{Introduction}

On peut très schématiquement distinguer deux grands types d'approche du langage :

1) le langage comme objet correspondant à un matériau verbal, un ensemble de formes intonées, agencées et organisées (constitutives des langues dans leur diversité) ;

2) le langage comme activité, relatif à des sujets qui pratiquent et manifestent cette activité (en production, en compréhension), activité qui parait spécifique de l'espèce humaine et qui correspond en tout cas à une activité fondamentale de l'homme.

Or dans la plupart des cas, un clivage se forme entre ces deux approches (cf. en particulier le clivage linguistique / cognition ou encore linguistique / pragmatique). Un tel clivage se fonde sur une conception des formes comme des « moyens » utilisés par des sujets parlant.

Notre approche ${ }^{1}$ vise au contraire à ne pas séparer les formes des sujets : il s'agit 
non pas de sujets qui utilisent des formes, mais de formes qui marquent et même construisent la présence et tracent l'activité de sujets (sous le mode particulier que leur confèrent ces formes). Les sujets présents sous ces différents modes n'ont rien d'hétérogène ou de transcendant aux formes, ils en constituent le produit même. Il s'agit donc d'aboutir à une théorie de ces formes en tant qu'elles construisent de façon multiple des positions inter-énonciatives, des modes d'assertion, d'interrogation, d'injonction, d'exclamation, de concession, etc. L'activité de langage à laquelle on s'intéresse est donc toute entière définie par ce qu'en tracent les formes, leur agencement et les contraintes que manifestent ces agencements. N'est pris en compte dans cette activité que ce que les formes permettent d'en dire.

\section{1. Énonciation}

On peut définir l'énonciation comme mettant en jeu, de façon indissociable trois processus, les «trois $\mathrm{R} »$ mis en avant par Culioli $^{2}$, dont la théorie inspire cet article : représentation, régulation, référenciation.

\section{1) Représentation}

Les opérations de représentation jouent elles-mêmes sur trois niveaux :

- niveau 1 : niveau du cognitif, des affects, des déterminations anthropologiques, physicoculturelles (niveau infra verbal);

- niveau 2 : niveau des agencements de formes qui font affleurer de façon spécifique le niveau 1 dont ils ne sont pas un simple reflet, un simple redoublement, une simple mise en forme: le matériau verbal est trace de construction de sens et de connaissance ;

- niveau 3: niveau hétérogène des représentations métalinguistiques ou épilinguistiques ${ }^{3}$, lieu d'une analyse de la langue; lieu de production de gloses ; lieu aussi de formalisations, partant du principe que le langage fournit des emplois possibles de ses formes à des fins métalinguistiques. Une formalisation est possible dès lors qu’il y a raisonnement, abstraction, va-et-vient permanent entre l'empirique et le formel. L'empirique consistant en la prise en compte des données de langue sans mettre à l'écart toutes celles qui résistent à leur intégration à un cadre formel préétabli et parfois exporté de domaines non fondés à partir de l'observation de ce type de données.

Cette articulation entre les trois niveaux fonde une approche non instrumentale du langage. Le langage n'est pas un instrument au service d'une pensée qui lui préexisterait, n'est pas une traduction ni un code qui permettrait de passer de la pensée au verbe (à supposer que l'on puisse savoir ce qu'est au juste la pensée). Le langage est constitutif d'une forme de pensée (parmi d'autres). Il construit des représentations mentales d'un

1 Cet article est le fruit d'un travail collectif qui doit l'essentiel aux travaux de Antoine Culioli, Sarah de Vogüé et Denis Paillard.

Cf. en particulier Culioli $(1990,1999)$.

Sur la notion d'épilinguistique, cf. en particulier De Vogüé (2001). 
certain ordre, que l'on appréhende comme étant le «sens » (non univoque, non stabilisé, non fini) de ce que nous disons et de ce que nous comprenons quand nous parlons, écrivons, lisons, interprétons.

\section{2) Référenciation}

Il s'agit des opérations par lesquelles le langage permet de dire quelque chose du monde (entendu au sens large, y compris au monde des sensations, des rêves, des imaginations, etc.), de le rendre partie intégrante d'un vouloir dire (vouloir dire sur le monde / vouloir dire du monde). Â travers les formes s'exprime quelque chose à propos d'un état de choses qui est - comme le saisit très bien le terme anglais de aboutness - ce dont on parle en disant ce qu'on dit. Les formes agencées qui constituent la matérialité des langues sont des formes en prise sur le monde. La référenciation est une construction, le monde en tant qu'il est dit de la façon dont il est dit, le lieu des rapports entre le dicible et l'indicible (ce dernier n'ayant de statut qu'à travers ce qui en est dit), ainsi que le lieu nous y reviendrons et ce sera le cœur de notre exposé - où s'exprime sous toutes sortes de façons possible (souffrance, échec, créativité, recours poétique, reformulation, bafouillement, recours à d'autres formes de référenciation) l'irréductible écart entre les deux (le dicible et l'indicible, le dire et le vouloir dire). Référenciation pose une référence qui est toujours construite; et qui est toujours aussi constitutive d'un reste : on n'épuise jamais le monde en le décrivant (comme s'en est amusé par exemple Georges Perec dans Tentative d'épuisement d'un lieu parisien); et en même temps, on peut le décrire à travers des milliards "d'exercices de styles » (comme s'en est amusé non moins élégamment Raymond Queneau).

\section{3) Régulation}

L'énonciation met en jeu des relations inter-énonciateurs ou plus précisément des points de vue, qui sont des positions énonciatives. Des positions énonciatives qui, comme on l'a dit, sont non pas des positions d'individus parlant dans leur singularité d'individus, mais des positions établies et marquées de façon organisée, structurée, par des formes agencées dans la langue. Par exemple par toutes les marques permettant de construire des formes d'intimation, d'injonction, de concession, en particulier par ce que l'on appelle «les mots du discours».

Si on appelle So la position de l'énonciateur et S'o celle du co-énonciateur telles qu'elles se construisent dans les énoncés, on peut établir qu'entre So et S'o les marques agencées des langues établissent des formes d'altérité aussi variables que complexes, jamais réductibles à ce qu'en saisissent de façon nivelante de grandes catégories grammaticales (comme celle de l'injonction, de l'interrogation, du souhait, etc.). Il faut en particulier souligner que S'o peut, sous des formes verbales multiples, se manifester d'une part comme l'image que So se fait de l'autre et d'autre part comme la représentation que se fait So de lui-même en tant qu'autre. La structuration des types d'altérité qui se dégage de l'étude des formes, il faut y insister, n'est pas réductible à celle des altérités que l'on peut établir (d'un point de vue sociologique, anthropologique, psychologique, etc.) entre des individus (même si, via le niveau 1, elles n'en sont pas non plus indépendantes). 
Jean-Jacques Franckel

Venons-en maintenant à un aspect central de l'énonciation : l'énonciation comme lieu et processus de prolifération et, quatrième $\mathbf{R}$ en jeu, de reformulation.

\section{Glose, reformulation, activité épilinguistique}

\subsection{Deux types de reformulation}

On peut distinguer au moins deux types de reformulation :

- l'une qui ressortit à une activité purement langagière, celle par laquelle on revient sur ce qui vient d'être dit, dans une tentative de cerner plus étroitement le vouloir dire en jeu ${ }^{4}$. Il peut s'agir du dire du locuteur lui-même cherchant à mieux se faire comprendre (ou à mieux comprendre lui-même ce qu'il veut dire) ou du dire de l'interlocuteur, dont on reformule le propos afin de s'assurer sinon de sa bonne compréhension, du moins de ce qu'un malentendu irréductible ne s'installe pas. Cette activité met en jeu de façon emblématique I mean en anglais ou, en français, des expressions telles que je veux dire..., que veux-tu dire au juste? ainsi que le maillage des mots du discours (en quelque sorte, d'une certaine façon, effectivement, etc.). Cette activité de reformulation s'inscrit dans le va-et-vient permanent entre dire et vouloir dire qui se nourrissent mutuellement.

- l'autre ressortit à une activité épilinguistique à finalité plus ou moins «explicative », consistant en une tentative d'expliciter le sens d'un mot, d'une expression, d'un énoncé, d'un texte. Pour avoir un accès matérialisable au sens d'un mot, d'une phrase, d'un texte, pour analyser ce sens, il faut le reformuler, donc aussi, par là même, le déplacer, le transformer, l'altérer. Le sens apparaît donc finalement comme une sorte de «point aveugle » : il n'a de statut qu'en tant que cerné mais non pointé, jamais touché en quelque sorte. Ce n'est pas le thème dont les différentes matérialisations, inscrites dans des rapports de synonymie ou de paraphrase seraient des variations, car en fait, tout est variation. Et l'on n'analyse (du moins le devrait-on) une forme à travers son rapprochement à une autre qu'à partir de l'écart plus ou moins explicitable que ce rapprochement permet de mettre en lumière et qui devient à son tour lieu nécessaire d'une nouvelle explicitation. Comme on le sait bien, mais comme aussi il convient de le rappeler, le sens est affaire de circulation, à la fois inépuisable et stabilisable (avec tout le problème de cerner ce qui constitue cette stabilisation).

\subsection{Dire et vouloir dire}

Le propre du dire ${ }^{5}$ est d'être à la fois constitutif d'un à dire ou d'un vouloir dire et en même temps toujours aussi constitutif d'un écart permanent ou presque entre dire et vouloir dire. S'agissant de référenciation, le rapport du dire au à dire débouche toujours sur un reste. Ce qui explicite n'épuise pas. Insistons : le vouloir dire n'est pas le préalable dont le dire serait la concrétisation : c'est le dire qui donne corps au vouloir dire et à l'écart qui s'ensuit. Cet écart se manifeste en particulier par une expression qui se cherche, se

$4 \quad$ Sur ce point mais avec d'autres problématiques, cf. en particulier les travaux de Jacqueline Authier-Revuz (1995, 2002 et 2012), ou Le Bot, M.-C., Schuwer, M., Richard, E (eds) (2008).

5 Pour une analyse du verbe dire, cf. Franckel, J.-J. (2015). 
redéploie, du bafouillement à la fuite en avant de la reformulation perpétuelle, ou encore se condense dans la difficulté de l'écriture ou la densité de la poésie.

On peut considérer un énoncé comme à la fois source et conséquence de trois types de vouloir dire:

- Vouloir dire des sujets (inscrit en particulier dans les processus de régulation). Il s'agit de ce qui dans un énoncé cherche à se dire de la part / du point de vue d'un sujet. Ce vouloir dire est accessible à travers ce qu'il (le sujet) dit et ce qu'il redéploie comme vouloir dire à travers même ce qu'il dit. Nous y avons suffisamment insisté : le vouloir dire n'est pas moins source que conséquence du dire ;

- Vouloir dire des mots (inscrit en particulier dans les processus de représentation). Ce vouloir dire ressortit à l'impossibilité de stabiliser le sens d'un mot, celui-ci échappant toujours aux tentatives de réduction à une définition stabilisée, comme on l'a souligné. En même temps ces sens ne sont pas aléatoires : chaque mot a des propriétés spécifiques, une identité qui lui permet de structurer ce vouloir dire, de l'inscrire dans des processus réguliers de stabilisation (représentation étant liée à régulation et référenciation);

- Vouloir dire du monde (inscrit en particulier dans les processus de référenciation). Le monde relève à la fois de l'indicible et du à dire dès lors qu'on en dit quelque chose, qu'on l'appréhende à travers des formes verbales: Tiens, la porte est ouverte! Qu'est-ce que ça vent dire? Ce vouloir dire du monde, qui est d'ailleurs aussi un vouloir dire du corps (le corps qui « s'exprime »), est à mettre en relation avec une sémiologie, où toutes les choses peuvent devenir des indices, des éléments «à interpréter», qu'il faut savoir « faire parler » ou auxquels il suffisait d'être attentif pour les entendre (comme l'illustre le travail du détective, du botaniste, de l'observateur avisé, et pourquoi pas, du linguiste se donnant la peine d'analyser un corpus).

Ainsi va le langage: les mots veulent dire, signifient; nous autres humains cherchons à dire de façon plus ou moins satisfaisante des choses plus ou moins compliquées, notamment dans le travail de l'écriture, avec ces mots, dont le travail du poète et du philosophe, entre autres ou du linguiste, parfois, montre que ce qu'ils cherchent à dire ne cesse de nous échapper en partie ; et cela à propos d'un monde, d'un corps de phénomènes, d'expériences et de sensations que l'on peut investir de sens, qui peuvent s'incorporer à une sémiotique, qui s'inscrivent dans ce déploiement foisonnant et incessant du langage.

\section{Une étude de cas}

Nous proposons d'illustrer de façon très minimale un aspect de ce qui précède par un exemple, qui mettra en avant, plutôt que la reformulation elle-même, la question de la prolifération des formes - les deux phénomènes étant indissociables autour de sens qui se perçoivent sinon comme identiques du moins comme très voisins. Il s'agit notamment de mettre en avant la singularité irréductible de chacune de ces formes (notamment à travers les contraintes mises en jeu par leur emploi) alors 
même que leur sens est parfois si proche que la différence ne peut le plus souvent être explicitée en première analyse (et même au-delà).

La possibilité de dire «la même chose » de plusieurs façons trouve sans doute son exemplification la plus immédiate dans la notion de synonymie, dont il a été montré qu'elle ne correspond jamais à une pure équivalence. Mais l'activité de reformulation dépasse très largement la mise en œuvre de synonymes.

\subsection{Corpus}

Considérons quelques-unes des réponses possibles à une question ${ }^{6}$ des plus banales, telle que :

- Tu crois qu'il sait conduire ce genre d'engin?

Nous entendons « réponse » en un sens délibérément très ouvert, afin d'illustrer de façon la plus large possible la notion de prolifération. L'important est que puisse être aisément perçue la proximité de valeur des différentes formes mobilisées dont chacune relève d'un fonctionnement propre, qu'il faudrait étudier dans le détail de sa spécificité.

\section{1) Dynamique positive de la réponse}

a) (Ben) Bien sûr (que oui, qu'il sait)! (₹ sûrement); Bien entendu (?que oui ; qu'il sait)! Naturellement! (?que oui ; qu'il sait); Évidemment! (?que oui; qu'il sait); Ca va de soi! Forcément! C'est la moindre des choses (qu'il sache); Et comment! Encore heureux! Heureusement! Quand même! ; Un peu, qu'il sait!

b) (Ben) Et puis quoi encore! Manquerait plus que ça (qu'il ne sache pas)! Ce serait le comble! Qu'est-ce que tu crois / imagines! Il ferait beau voir (qu'il ne sache pas)! Comment ne saurait-il pas? (alors qu'il a déjà réalisé plusieurs chantiers); Laisse tomber (c'est un virtuose de la pelleteuse)!; Tu penses bien! Je pense bien!

c) J'en suis absolument certain, sûr, convaincu, persuadé.

Cette répartition ne constitue pas une typologie des réponses mais permet de dessiner sommairement trois modes (sans frontière stricte) :

a) caractère évident de la réponse positive quant au fait de savoir. Il importe d'insister sur le fait qu'il ne s'agit pas d'un commentaire de oui, mais de dire à quel titre (au titre du naturel, de la force des choses, etc.) la réponse positive s'impose ;

b) remise en cause de la légitimité même de la question : la réponse marque en même temps l'absurdité d'une réponse négative qui du coup disqualifie l'alternative (la disqualification de l'illégitime introduisant la problématique du légitime / illégitime qui n'est nullement inscrite dans la forme même de la question);

c) réponse positive établie avec des marqueurs de conviction.

\section{2) Dynamique négative}

Tu rêves! Tu n’y penses pas! Penses-tu! (comparer avec Tu penses! Tu penses bien! Je pense bien !) ; Tu n'y songes pas (*Tu songes! / *Tu n'y rêves pas !) ; Justement pas ; Justement : c'est 
Prolifération et reformulation comme traces de l'activité énonciative à travers l'agencement des formes

là que ça coince. Voilà le bic! C'est bien le problème ; Ca m'étonnerait; Ce serait trop beau; Ca ne risque pas; Sûrement pas; Certainement pas.

\section{3) Non tranché}

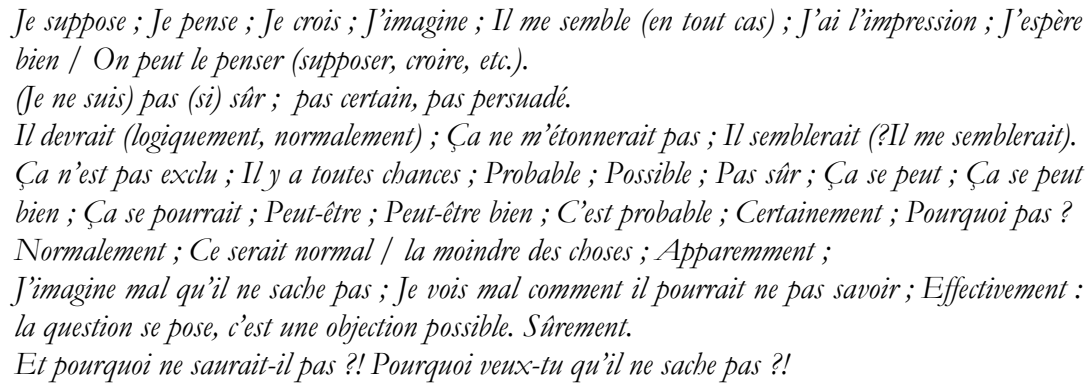

\subsection{Commentaires}

Confronté à la richesse d'un tel corpus (dans son apparente banalité), non limitatif, on se limitera ici à quelques commentaires.

3.2.1. La prolifération elle-même fait phénomène. Que signifie cette profusion de formes? De quel phénomène cognitif est-elle le symptôme ? Nous défendons 1) que ce ne sont pas des variations d'un même qui serait formulable ;2) que cette prolifération n'est pas simplement affaire de pragmatique, qu'elle met en jeu à part entière et dans toutes leur spécificités les propriétés des formes agencées et intonées de la langue.

3.2.2. Nous tenons en effet qu'il s'agit de formes radicalement différentes avec des sens profondément différents, quelle que soit la proximité intuitive des gloses par lesquelles on peut spontanément tenter de les expliciter et la difficulté de préciser la différence.

Prenons un exemple ${ }^{7}$. On peut montrer que bien entendu et naturellement relèvent de logiques profondément différentes : naturellement marque que c'est au titre et au nom du naturel que la réponse donnée est validée; bien entendu introduit un point de vue qui pose un autre point de vue (celui qui correspondrait à l'autre branche de l'alternative) comme relevant du malentendu. Il s'agit de deux logiques profondément différentes pour un sens très voisin.

On pourrait de même analyser les différences profondes entre je crois et je pense qui paraissent pourtant très voisins comme réponse positive non certaine. On a affaire à des croisements locaux qui brouillent ces différences. Car par ailleurs croire et penser n'ont rien à voir ${ }^{8}$. Pour ne prendre qu'un exemple, on peut observer la différence de comportement entre Je crois bien (Il me semble, Je dirais que oui sauf erreur, avec une intonation assertive) et Je pense bien! (Et comment! avec une intonation exclamative). En outre, Je pense bien! est proche de Tu penses bien! alors que Tu crois bien! n'a nullement le même sens que Je crois bien! Soulignons que l'intonation est déterminée par la forme

\footnotetext{
Cf. sur ce point les travaux de Vladimirska $(2008,2009)$.

Cf. ici-même sur croire et Culioli aussi (1990, p. 204-205).
} 
même de la séquence, les deux schémas intonatifs ne pouvant être intervertis. Nous reviendrons en 3.2.6. sur l'importance de ce type de contraintes dont l'analyse fine supposerait un travail spécifique approfondi sur chacune des formes.

3.2.3. Ces formes ne constituent pas un «commentaire» ou des variantes de oui ou non (beureusement ne signifie pas : oui et c'est beureux). Ces formes disent autre chose que oui ou non, et pour certaines disent à quel titre et dans quelle mesure la réponse qui est donnée est donnée. Par exemple avec forcément, non seulement on asserte qu'il sait, mais on asserte que la force des choses est à l'œuvre, et à l'œuvre de façon complexe à la fois dans le dire de la réponse et dans le fait même (qu'il sait conduire).

3.2.4. La lecture du corpus montre bien souvent la difficulté de déterminer de quoi il est (ou il est fait) question dans la réponse : celle-ci travaille la question, on pourrait presque dire la pose. C'est en particulier le cas lorsque la réponse introduit un point de vue qui constitue alors la question comme un premier point de vue relativement auquel peuvent se nouer toutes sortes de rapport d'altérité (la réponse pouvant établir un point de vue où la question ne se pose pas).

La réponse peut encore être donnée comme s'imposant d'elle-même comme dans l'exemple: Manquerait plus que ça! Nous attirons l'attention sur la complexité de l'analyse d'une telle réponse : que manquerait-il au juste ? Il s'agit de ça, dira-t-on, mais il faut établir le raisonnement par lequel ça renvoie à qu'il ne sache pas (glose introduisant donc le subjonctif et la forme négative du verbe savoir introduit au présent indicatif dans la question) : c'est la branche négative de l'alternative introduite par la question qui est à la fois posée comme fictive (cas limite qu'on refuse d'envisager) et comme susceptible de «manquer». Mais reste encore à établir à quoi ne pas savoir manquerait : ce serait le comble, mais que s'agit-il au juste de combler? Dans Manquerait plus que ça! reste enfin à régler le statut de (ne) plus qui fait entendre qu'il y a des choses (lesquelles ?) qui manquent déjà.

3.2.5. Les questions posées par l'exemple précédent doivent l'être pour toutes les formes dans un programme d'analyse proprement linguistique, mettant en jeu le raisonnement le plus rigoureux possible. L'aspect cumulatif du travail, encore très incertain, consisterait à reprendre des raisonnements amorcés pour tenter de les pousser plus loin, de façon à la fois plus précise et plus généralisable. Il apparaît que les considérations proprement pragmatiques susceptibles de prendre en compte les effets de sens mis en évidence dans l'échantillon des réponses proposées tendent à faire l'économie de ce type de raisonnement qui conduit à observer et parfois à découvrir des données de plus en fines. On ne peut se contenter de considérer que ces formes sont les variantes plus ou moins «figées », plus ou moins emphatiques d'un même type de réponse (positif ou négatif) à la question.

Nous évoquons quelques terrains d'analyse (vierges ou en friche) restant à creuser à titre d'exemples :

- A quoi renvoie quoi dans Et puis quoi encore?

- Que signifie si dans Pas si sûr? Cf. ??Pas tellement sûr alors que dans Je ne suis pas si sûr, si commute avec tellement sans grande difficulté ; 

trompe pas)?

- Quelle est au juste la valeur de devoir dans Il devrait savoir (normalement, si je ne me

- Que signifie aller de soi?

- Que signifie beau dans : Il ferait beau voir?

- Que signifie croire dans Qu'est-ce que tu crois! (parenté possible avec Qu'est-ce que tu imagines, qui disparaît dans la plupart des emplois de croire, à commencer par celui de la question)?

3.2.6. Ce programme d'analyse linguistique du fonctionnement des formes mises en jeu dans ce foisonnement de réponses passe par la prise en compte des contraintes qui déterminent ce fonctionnement. Nous en avons déjà donné quelques exemples (cf. 3.2.2.). Ces contraintes concernent l'emploi des formes, y compris dans des aspects qui tendent à passer au second plan dans une analyse proprement syntaxique, en particulier le positionnement et l'intonation.

S'agissant du positionnement, on peut prendre l'exemple de la différence essentielle qui apparaît entre les deux réponses suivantes :

- Forcément! il a son CAP de conducteur d'engin : la réponse asserte qu'il s'agit d'une donnée établie :

- Il a forcément son CAP de conducteur d'engin: la réponse marque une supposition (il doit bien / forcément avoir son $(A P)$.

S'agissant de l'intonation, il s'agit là aussi d'un terrain d'une extrême complexité, dont nous donnons un petit échantillon (en dehors des exemples déjà mentionnés d'intonation contrainte avec les verbes croire et penser) :

- À côté de Et comment! parfaitement naturel, on observe le caractère discordant de *Ah comment! ( $\neq$ Ah (bon) ?!); *Oh comment!;

- On peut observer la différence entre Oh oui! et $A h$ oui! (avec des intonations distinctes) :

- Oh oui ! va dans le sens de la question et d'une réponse positive à cette question : Je pense, Il ne devrait pas y avoir de problème ;

- Ah oui ! est plus difficilement interprétable et plus complexe : il peut marquer le fait même que la question est à prendre en compte (Ah oui! c'est vrai c'est une bonne question, je n'y avais pas pensé, avec la question, là encore, de déterminer ce qui dans l'affaire est posé comme vrai).

3.2.7. Enfin, il peut être intéressant d'un point de vue cognitif de souligner que la plupart de ces contraintes ne sont pas visibles. Elles passent inaperçues, nous n'en avons pas conscience sauf à nous livrer à ce qui est supposé constituer le fondement même du travail du linguiste: débusquer les contraintes propres à alimenter un raisonnement rigoureux dans la recherche du fonctionnement des formes et de leur organisation. Lorsque ces contraintes sont lâches, elles laissent place à ce qui va pouvoir être repéré ou catégorisé comme fautif, et deviennent par là-même repérables. En revanche, la résistance des contraintes les plus profondes à leur transgressibilité tend à les rendre muettes: on peut considérer que l'impossibilité de $\mathrm{Ob}$ comment! comme alternative à Et comment! dans l'exemple considéré, ou de Tu crois bien! comme alternative à Tu penses bien! ou de Crois-tu! comme alternative à Penses-tu! n'est pas 
Jean-Jacques Franckel

immédiatement décelable. On peut avancer que plus une contrainte est profonde, moins elle est visible; que la conscience que l'on peut avoir des contraintes est inversement proportionnelle à l'importance de leur rôle dans la cohésion structurante de la langue.

\section{Conclusion}

L’exemple proposé peut être considéré comme une manière d'illustration des $3 \mathbf{R}$ constitutifs de l'énonciation, avec des pondérations variables sur chacun :

- les différentes façons dont la réponse permet de travailler la question (et peut notamment dans certains cas remettre en cause son bien-fondé) mettent en jeu de façon prépondérante régulation et représentation;

- la façon dont la réponse s'ancre sur un état de choses met en jeu la référenciation;

- enfin toute l'activité épilinguistique en jeu dans le foisonnement des reformulations relève plus spécifiquement du niveau 3 de la représentation.

Le travail du linguiste s'ancre sur l'observation des formes et des contraintes qui régissent leur organisation, dans un constant va-et-vient entre l'empirique et le formel donnant peu à peu accès au fonctionnement cognitif dont formes et contraintes sont une trace, le langage étant une forme d'activité cognitive parmi d'autres.

Nous soutenons contre nombre de théories cognitives dominantes que le langage ne saurait trouver ses raisons dans quelque substrat sémantico-cognitif autonome qu'il se contenterait d'exprimer, mais qu'il est lui-même activité de construction de sens et de connaissance. C'est le sens construit par les énoncés qui nous importe, notre analyse part des formes linguistiques et des formes seules, pour considérer que le sens qui s'y construit est un sens non pas préétabli, mais au contraire inconnu et à découvrir, dont il s'agit de dégager la spécificité. 
Prolifération et reformulation comme traces de l'activité énonciative à travers l'agencement des formes

\section{Bibliographie}

AUTHIER-REVUZ J., 1995, Ces mots qui ne vont pas de soi : boucles réflexives et non-coïncidences du dire, $\mathrm{t}$. 1 et 2, Paris, Larousse.

Authier-Revuz, J., Lala, M.-C. (eds), 2002, Figures d'ajout. Phrase, texte, écriture, Paris, Presses Sorbonne Nouvelle.

Authier-Revuz, J. et Doquet, C., 2012, “'Ce que je veux dire...’ - Accompagnements métadiscursifs d'une défaite de la parole», in C. Doquet, E. Richard (eds), Les représentations de l'oral chez. Lagarce, Édition Academia, p. 17-64.

CUlioli, A., 1990, « Representation, referential processes and regulation ; Language activity as form production and recognition» in Pour une linguistique de l'énonciation, t.1, Ophrys, p. 177-213.

Culioli, A., 1999, «Les modalités d'expression de la temporalité sont-elles révélatrices de spécificités culturelles ? » in Pour une linguistique de l'énonciation, t.2, Ophrys, p. 159-164.

De VogüÉ, S., PAILLARD, D.,1987, « Modes de présence de l'autre », in Les particules énonciatives en Russe contemporain, volume 2, collection ERA 642, republié et traduit en portugais dans l'ouvrage (2012) Linguagem et enunciac ão, representaç ã o e regulaç ã o, Editora Contexto, p. 131-159.

DE VogüÉ, S., 2001, «L'épilangue au pied de la lettre. A propos du concept grammatical de condition », Le gré des langues, 16, p. 9-48.

FRANCKEL, J.-J., 2015, « Dire », Langue Française, 186, Larousse, p. 87-102.

LE BOT, M.-C., SCHUwER, M., RiCHARD, E. (eds), 2008, La Reformulation. Marqueurs linguistiques - Stratégies énonciatives, Rennes, PUR, coll. "Rivages linguistiques".

PAILlard, D., 2002, «Les mots du discours: identité et variation», Cahiers de linguistique de I'INALCO, Paris, INALCO, p. 31-47.

PAILlaRD, D., 2010, « Marqueurs discursifs et scène énonciative » in S. Hancil (éd) Connecteurs discursifs, Presses Universitaires de Rouen, p. 13-39.

VLADIMIRSKA, E., 2008, «A propos de naturellement, bien entendu et bien sûr », Information Grammaticale, 119, p. 3-7.

VladimirSKA, E., 2011, «Les Mots du discours et l'opération de rectification : l'exemple de vraiment, pas vraiment et bien entendu» in La Rectification à l'oral et à l'écrit, Paris, Ophrys, collection «L'Homme dans la langue ». 
